\title{
Innover en entreprise : la solitude du coureur de fond
}

\section{Industrial innovators: the loneliness of the long distance runner ${ }^{1}$}

\author{
Michel Moruzzis ${ }^{1}$ \\ ${ }^{1}$ Michel Moruzzis Consulting, France, michel.moruzzis-consulting@orange.fr \\ Les opinions présentées dans cet article n'engagent que son auteur, même si elles s'appuient sur des exemples tirés \\ de son expérience professionnelle passée.
}

RÉSUMÉ. "Le rendement des idées est faible »; si ce constat résume l'état de l'innovation dans les entreprises industrielles françaises, il cache la réalité des difficultés des innovateurs. Au terme d'une carrière de presque quarante ans consacrée aux études amont, l'auteur voit effectivement l'innovation comme une course de fond, la plupart du temps en solitaire et soumise à de nombreux freins. Pour l'expliquer, l'auteur présente trois exemples tirés de sa propre expérience professionnelle et il en extrait une analyse plus générale des différents freins à l'innovation. De cette analyse, l'auteur conclut que pour se développer, une entreprise industrielle doit considérer l'innovation comme un élément de son ADN et que finalement c'est dans le domaine du management que les entreprises ont le plus besoin d'innovation. Si cette analyse peut inciter les entreprises industrielles à pratiquer les sports d'équipe pour innover, alors ce témoignage aura atteint son objectif.

ABSTRACT. To sum up the state of innovation in French industries, it could be said that "Smart ideas give poor returns". After almost forty years spent in advanced studies, the author realized that industrial innovators must be tough long distance runners, and that many obstacles are paving their way. Then he thought that his experience could be used to highlight and explain this point of view. Starting from the description of three cases from his own work, the author attempts to describe more general obstacles which are probably affecting many industrial companies. Finally, the author concludes that innovation in French industrial companies should be considered more seriously and as a part of the company's DNA. He also believes that management is probably the domain in which companies really need innovation. As a conclusion, the author would be delighted should this analysis stimulate companies to much more practice team sports to innovate.

MOTS-CLÉS. Invention et innovation, innovation et entreprise, peur d'innover, difficulté à innover, freins à l'innovation, gouvernance de l'innovation, management innovant.

KEYWORDS. Invention and innovation, innovation and industrial companies, obstacles to innovation, fear of innovation, management of innovation, innovative management.

\section{Introduction}

Parlant d'innovation dans l'entreprise, un ancien collègue de l'auteur (qui se reconnaîtra s'il lit ces lignes) résumait souvent la situation de la manière suivante : « Le rendement des idées est faible ».

Après presque quarante années consacrées aux études amont (voir 3.3.1) dans le milieu industriel, l'auteur est arrivé à la conclusion qu'il ne s'agissait pas d'une boutade (malheureusement !). Il a alors tenté d'y voir un peu plus clair, d'essayer de comprendre quels sont les freins à l'innovation technique dans les entreprises industrielles, et d'imaginer quelles seraient les pistes pour améliorer la situation.

Heureusement, des cas concrets vont illustrer cette analyse. Ils concernent trois exemples représentatifs de par leur ampleur (il s'agit en effet de travaux couvrant plusieurs années voire plusieurs décennies), leur diversité, et aussi parce qu'au-delà des aspects techniques, ils ont des liens avec d'autres domaines d'activité internes à l'entreprise et qu'ils font intervenir des interlocuteurs extérieurs.

Cette analyse constitue un témoignage personnel qui s'appuie sur une étude de cas vécus. L'auteur tente de déduire de sa propre expérience des enseignements plus généraux, en s'appuyant notamment sur des échanges qu'il a pu avoir à l'extérieur de son entreprise.

1. « The loneliness of the long distance runner » est un film britannique réalisé par Tony Richardson (1962), selon la nouvelle d'Alan Sillitoe (1959).

(c) 2016 ISTE OpenScience - Published by ISTE Ltd. London, UK - openscience.fr 
Mais avant d'aller plus loin, rappelons quelques définitions :

- Inventer : «Réaliser quelque chose de nouveau, à quoi personne n'avait pensé auparavant, que personne n'avait fait. »;

- Innover : «Introduire quelque chose de nouveau pour remplacer quelque chose d'ancien dans un domaine quelconque. ».

Dans l'entreprise, l'auteur a été inventeur et innovateur. Inventeur lorsqu'il a trouvé quelque chose de nouveau, par exemple au cours d'une étude, et innovateur lorsqu'il a contribué à développer (ce qui signifie ici, définir, concevoir, réaliser et mettre en œuvre dans un équipement) une de ses inventions. C'est son rôle en tant qu'innovateur qui est le plus utile pour le sujet qui nous intéresse dans cet article.

Il ne s'agit bien entendu que d'opinions personnelles sur la question et le propos est surtout d'expliquer le point de vue de l'auteur sur les difficultés qu'un individu rencontre pour innover dans son entreprise. Mais comme, selon lui, on retrouve des difficultés de même nature au niveau d'une entreprise lorsque celle-ci cherche à innover au sein d'une communauté plus vaste, cette analyse peut revêtir un caractère (et probablement aussi un intérêt) plus général.

Dans ce qui suit, l'analyse est présentée en trois étapes :

- La première étape est une étude de cas, portant sur trois exemples dont le fil conducteur est la complexification des interactions mises en jeu lorsque le périmètre d'influence de l'objet de l'invention croît de l'interservices à l'inter-domaines d'une même entreprise, puis de l'intra-entreprise à l'interentreprises. En effet, une des hypothèses que l'auteur souhaite développer est que les freins à l'innovation croissent avec la taille du système des parties prenantes ;

- La deuxième étape consiste en l'analyse des freins à l'innovation que l'auteur tente de généraliser à partir des cas d'étude considérés comme des échantillons ;

- La troisième étape tente de proposer, à titre de perspectives, des pistes pour améliorer la situation du coureur de fond solitaire.

\section{Trois exemples en quelques mots}

Comme les trois exemples sont issus du domaine des radars, il est sans doute bon d'en dire deux mots.

Le sigle anglais RADAR (RAdio Detection And Ranging) désigne un appareil destiné à détecter et localiser des objets grâce aux ondes électromagnétiques.

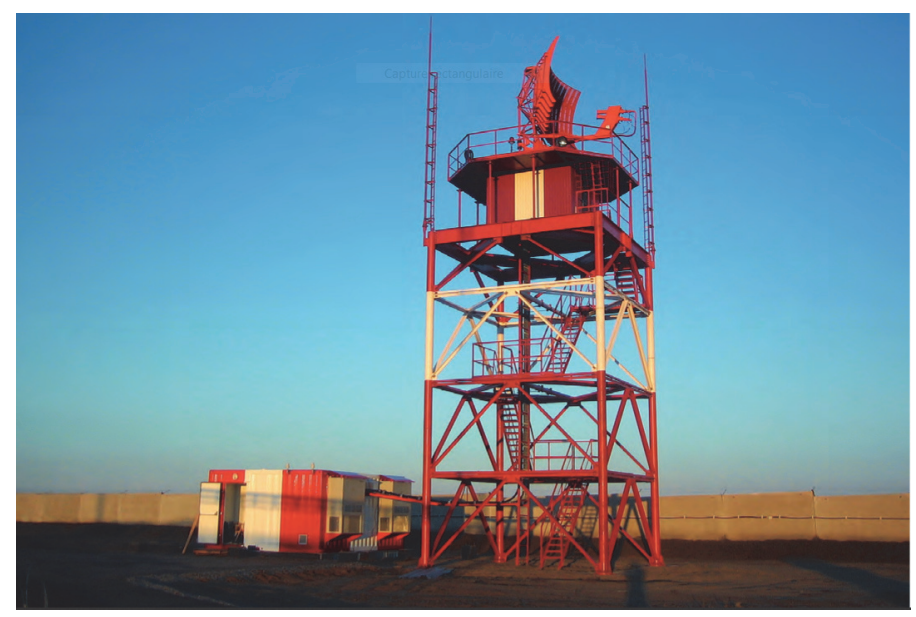

Figure 1. Radar typique (STAR 2000 de Thales) - (Source : société Thales) 
Cet appareil comporte classiquement un émetteur, une antenne, un récepteur et un système d'exploitation. Le radar utilise la propriété qu'ont les ondes électromagnétiques à se réfléchir sur tout obstacle, créant ainsi une onde de retour susceptible d'être décelée par un récepteur adapté à ce signal (Darricau 2015), (Billaud, et al. 2014).

La figure ci-dessous illustre un radar typique destiné au contrôle du trafic aérien civil.

Les exemples abordés dans cet article concernent des travaux d'études qui ont été menés par l'auteur au cours des quarante dernières années et qui ont donné lieu à plusieurs publications et brevets ${ }^{2}$. On ne parle ici que des travaux réalisés pendant cette période car il s'agit de témoignages vécus par l'auteur, mais pour la plupart, des travaux antérieurs avaient bien entendu été conduits par d'autres.

Les trois exemples ont été choisis notamment parce qu'ils permettent d'aborder des niveaux croissants d'interaction :

- le premier exemple (NCTR - Non Cooperative Target Recognition -) met en œuvre différentes spécialités techniques au sein de l'entreprise ;

- le deuxième (MSPSR - Multi Static Primary Surveillance Radar -) adresse différentes fonctions (autre que techniques) mais toujours dans l'entreprise ;

- le troisième (éoliennes) met en lumière les liens entre l'entreprise et ses interlocuteurs extérieurs.

\subsection{Exemple 1 : NCTR}

\subsubsection{De quoi s'agit-il ?}

La reconnaissance de cibles non-coopératives (en anglais Non Cooperative Target Recognition) est une fonction permettant à un capteur, en l'occurrence un radar, non seulement de « détecter » la présence d'un objet, appelé « cible » dans le langage des radaristes, mais de « reconnaître » ou « classifier » sa nature, sans que cette opération ne nécessite une coopération de la part de la cible.

La cible en question peut être un aéronef, dont on cherche la classe (par exemple chasseur, avion de ligne, hélicoptère...) à partir de plusieurs attributs (par exemple la taille, la vitesse, l'altitude, etc...).

Sans trop entrer dans les détails, le lecteur pourra retenir que la mise au point de cette fonction repose sur trois éléments :

- des algorithmes de traitement permettant d'extraire les informations intéressantes (ou attributs);

- une base de données renfermant les caractéristiques de cibles existantes rangées par classes, base à laquelle on compare les attributs extraits, ce qui permet d'élaborer une information synthétique relative à la classe de l'objet ;

- une méthodologie permettant d'exprimer les performances de la fonction (par exemple, la probabilité de fournir la classe correcte) et de les évaluer.

On ne peut pas réellement identifier une date ou une époque signant les origines de ces travaux, car ils sont intimement liés au développement du radar lui-même. Pour se concentrer sur une période assez récente, on peut toutefois faire référence à (Delhote et Moruzzis 1998), (Moruzzis et Colin 1998b), (Moruzzis, Saulais, et al. 2004), (Boulay, et al. 2011), (Moruzzis, Innovation radar et développement éolien 2011) pour les traitements, algorithmes et performances et à (Moruzzis, Guillerot et Lestrade 1998a), (Delhote et Moruzzis 1998), (Van der Heiden 1998), (Tait 2005), pour les notions de bases de données.

2. Les références aux brevets et publications les plus significatifs pour illustrer cet article sont fournies dans la bibliographie. Ce n'est pas par excès d'égocentrisme que, dans cet article, l'auteur fait souvent référence à des travaux auxquels il a contribué, mais pour fournir des indications sur les orientations qu'il s'est efforcé de donner. 


\subsubsection{Quels sont les objectifs?}

On s'intéresse ici aux travaux qui ont été menés par l'auteur dans l'objectif de fournir une fonction opérationnelle aux utilisateurs finaux. Les travaux concernaient donc la mise au point de la fonction dans son ensemble, et ne se focalisaient pas sur un de ses aspects particuliers.

\subsubsection{Quels travaux ont été réalisés ?}

S'agissant de traitements radar et notamment ceux destinés aux fonctions de classification, il est courant que les travaux portent sur des éléments considérés comme « nobles » (par exemple des méthodes particulières de traitement du signal), mais leur poids dans la fonction globale n'est pas si important que cela, et l'apport d'une méthode par rapport à une autre est souvent marginal.

Il est apparu assez vite à l'auteur que cette manière d'aborder le sujet ne permettrait pas d'aboutir à la mise en œuvre d'une fonction opérationnelle. C'est pourquoi il a œuvré pour réorienter les travaux vers une démarche plus globale, ce qui a peu à peu conduit à formaliser une approche multi-domaine technique jugée nécessaire au développement de la fonction NCTR. En résumé, cette approche nécessite la mise en œuvre simultanée de plusieurs aspects :

- des compétences techniques pluridisciplinaires (analyse de signaux, traitements, bases de données, simulations, calculs de performances, développement) ;

- une vision à long terme et des actions à court terme (approche incrémentale) ;

- une synergie permanente entre les études et la réalité terrain.

Enfin, sans entrer trop dans les détails, l'auteur pense utile de mentionner l'importance de la modélisation, au sens de la représentation raisonnée et à bonne échelle du phénomène physique à étudier, qui est indispensable pour :

- l'aide à la définition des traitements et à leur mise au point ;

- la définition, l'évaluation et la prédiction des performances ;

- la constitution des bases de données opérationnelles.

\subsubsection{Quel est le bilan?}

A l'heure actuelle, plusieurs radars dans le monde sont munis de fonctions NCTR de premier niveau, c'est-à-dire capables de fournir la classe des cibles, mais le développement de la fonction à un niveau plus détaillé butte toujours sur la question des bases de données.

Cependant, si on voulait mettre en œuvre cette nouvelle fonction, on pourrait dès maintenant passer au développement d'une méthode de type incrémental. Une méthode qui n'est pourtant pas « nouvelle »; en effet, une vision de la fonction complète et des étapes nécessaires à son développement a été décrite il y a déjà vingt ans (Moruzzis, Guillerot et Lestrade 1998a), (Delhote et Moruzzis 1998), (Moruzzis et Colin 1998b). Dans cette approche du problème, le schéma de développement est incrémental ; la fonction peut être développée indépendamment des traitements et des outils utilisés pour constituer la base de données et l'exploitation de la fonction est faite en utilisant les meilleurs traitements et les meilleurs outils de modélisation existants. Ses performances s'améliorent dans le temps au rythme de l'évolution de ces traitements et outils, via des mises à jour.

Malheureusement le débat semble encore (ou à nouveau) porter sur la recherche de " nouveautés » dans certains domaines techniques notamment le traitement du signal, alors que les éléments nécessaires existent déjà, même s'ils peuvent (et devront continuer à) être améliorés. Par exemple le groupe Thales propose en 2016 un projet de thèse (Thales 2016) « Développement d'algorithmes innovants d'apprentissage et de reconnaissance de cibles non-coopératives à partir de signatures Radar micro-Doppler ». C'est d'ailleurs selon l'auteur une bonne illustration du complexe " scientifico-moderniste » que l'on aborde un peu plus loin. 
Cet exemple est intéressant car il permet d'illustrer plusieurs syndromes de l'innovation qui sont présentés plus loin (3.2.1). Il permet aussi d'évoquer une autre difficulté qu'ont les entreprises à structurer leur propre patrimoine technique, ce qui peut les entraîner à ignorer ou oublier leurs propres connaissances (3.2.3).

\subsection{Exemple 2 : MSPSR}

Cet exemple traite du cas d'un nouveau concept de radar (MSPSR : Multi-Static Primary Surveillance Radar). Il permet d'aborder les aspects relatifs à la manière d'accompagner la mise sur le marché d'un nouveau système : même si ce nouveau système présente de nombreux avantages pour le client, comment lui en apporter la preuve, et comment vaincre les réticences naturelles au changement ?

\subsubsection{De quoi s'agit-il ?}

L'image que le grand public se fait d'un radar est celle d'une grande antenne tournante et d'un écran sur lequel tourne une sorte de rayon vert qui laisse quelques traces là où sont les avions détectés. Cette image est bonne dans le cas général d'un radar «monostatique », c'est-à-dire dans lequel l'émetteur et le récepteur sont au même endroit. Mais il existe une autre catégorie de radars utilisant plusieurs émetteurs et/ou plusieurs récepteurs, localisés à différents endroits, et sans antenne tournante. On parle alors d'un radar « multistatique ». Le concept de radar multistatique est en fait aussi vieux que le radar lui-même puisque, déjà en 1904, le « Telemobilskop » de l'ingénieur allemand Christian Hülsmeyer, bien que ne mesurant pas la distance, fonctionnait sur le principe bistatique (Willis et Griffiths 2007). Plus tard, en 1935, c'est le système radar britannique « Chain Home », également bistatique (Willis et Griffiths 2007), qui joua un rôle décisif dans la bataille d'Angleterre et qui contribua par là-même à inverser le cours de la seconde guerre mondiale. Plus récemment, dans les années 80 , le démonstrateur RIAS (Dorey, Blanchard et Christophe 1984) a montré la faisabilité du concept bistatique moderne.

A l'heure actuelle, presque tous les radars sont monostatiques. Mais les progrès constants réalisés notamment en termes de puissance de calcul permettent maintenant, ce qui n'était pas le cas il y a encore quelques années, de réaliser des radars multistatiques. En effet, dans le cas des radars multistatiques, il faut en pratique mettre en œuvre des méthodes dites de FFC (Formation de Faisceau par le Calcul) ainsi que des méthodes de filtrage numérique assez sophistiquées pour supprimer certains effets néfastes comme le brouillage par le trajet direct entre l'émetteur et le récepteur. Ces traitements nécessitent de grandes puissances de calcul en temps réel, devenues maintenant accessibles.

C'est la raison pour laquelle il est devenu pertinent de poser la question de l'intérêt de développer cette technologie (pour en savoir un peu plus sur le concept MSPSR, le lecteur pourra se reporter par exemple aux documents (Moruzzis 2007) et (Moruzzis, Beauquet et Campoy 2010).

C'est en particulier dans le domaine des applications du contrôle du trafic aérien civil que le sujet est devenu d'actualité, et c'est ce sujet que l'on va analyser plus en détail dans ce qui suit.

\subsubsection{Quels sont les objectifs?}

Les deux principales questions que se pose la communauté ATM (Air Trafic Management) à ce sujet sont :

- le concept MSPSR est-il la bonne réponse pour assurer la fonction « Surveillance » ${ }^{3} \mathrm{du}$ contrôle du trafic aérien civil de demain, en particulier en termes de coût?

- si oui, comment et quand assurer la transition avec les équipements actuels (radar monostatiques) ?

3. Les trois fonctions principales du contrôle du trafic aérien civil sont dites « CNS » (Communication, Navigation, Surveillance). 


\subsubsection{Quels travaux ont été réalisés?}

Des travaux ont été réalisés ces dernières années sous la forme de démonstrateurs techniques, pour montrer la capacité réelle à maîtriser ce nouveau concept. En parallèle, des études de définition ont été conduites (voir par exemple (Moruzzis 2007) pour analyser son intérêt en termes de compromis coûtperformance, comparativement aux solutions classiques (radars monostatiques).

Ces travaux ont porté sur des aspects «classiques » (étude de concept, analyse de performance, coût, etc...), mais ils ont surtout mis en lumière le fait que, pour aborder correctement le sujet on ne pouvait pas se contenter de traiter les seuls aspects techniques, et que tous les métiers de l'entreprise étaient concernés.

En effet, la nouveauté du concept conduit à modifier plusieurs habitudes, ce qui fait que l'on ne peut pas traiter ce sujet simplement comme on le fait pour un nouveau radar classique. En l'occurrence, il s'agit de revisiter plusieurs métiers pour les adapter à de nouvelles méthodes :

- la fonction marketing (quel est le marché, quelle est sa maturité, comment accompagner les clients dans une transition qui peut être jugée brutale ?) ;

- les aspects commerciaux (quel est le bon prix, que vend-t-on, un produit ou un service ?) ;

- l'industrialisation et la production (comment modifier les habitudes de l'entreprise pour l'adapter à la production d'un objet aussi différent des radars habituels, quelle est la bonne structure « make/team/buy », comment et quand mettre en place une nouvelle chaîne de production ?) ;

- les aspects intégration, validation, qualification (comment définir les interfaces, comment adapter les moyens de validation et de qualification au nouveau concept ?) ;

- la logistique (comment aborder la maintenance d'un objet aussi particulier, les méthodes classiques de formation et de gestion de rechanges sont-elles toujours pertinentes, faut-il passer à des concepts de maintenance plus proches de ceux de la grande distribution ?) ;

- les aspects réglementaires (comment faire évoluer les normes de sureté ? que faire pour contribuer à leur évolution ?);

- la transition industrielle (comment gérer la transition monostatique/multistatique du parc mondial ? comment accompagner les clients dans cette transition?).

En particulier, une question reste ouverte concernant la manière d'aborder le marché. Il est en effet assez difficile d'anticiper une telle transition, et pour le faire correctement il semble indispensable d'entretenir des liens étroits avec l'ensemble des utilisateurs et de mettre à jour en permanence une étude de marché bien documentée.

Un des moyens qui a commencé à être mis en œuvre pour répondre à ce besoin a consisté à réaliser des études en coopération entre les industriels et les utilisateurs, notamment au titre du programme SESAR (Single European Sky ATM Research) (SESAR 2015) (SESAR 2016).

Le sujet reste bien sûr d'actualité ; par exemple Eurocontrol (l'organisation intergouvernementale européenne groupant 41 états membres et dont la mission est l'harmonisation de la navigation aérienne en Europe) a intégré les spécificités du MSPSR dans une mise à jour récente des spécifications de performance des systèmes de surveillance (Eurocontrol 2015) et des travaux de standardisation sont en cours chez ETSI (ETSI 2016).

\subsubsection{Quel est le bilan?}

Lors des études initiales du concept, les industriels envisageaient une production dans un délai de l'ordre de 6 à 7 ans (Moruzzis, Alternative Detection Technique to Supplement PSR Coverage (ADT) Final Report 2007). La vision en 2013-2014 des organismes internationaux (notamment l'OACI 
et Eurocontrol) est synthétisée sous la forme de « roadmaps » telles que celles présentées sur la Figure 2 ci-dessous (OACI à gauche, Eurocontrol à droite). Elles font bien apparaître cette nouvelle technologie MSPSR, et envisagent son déploiement à partir de 2020 et au-delà.
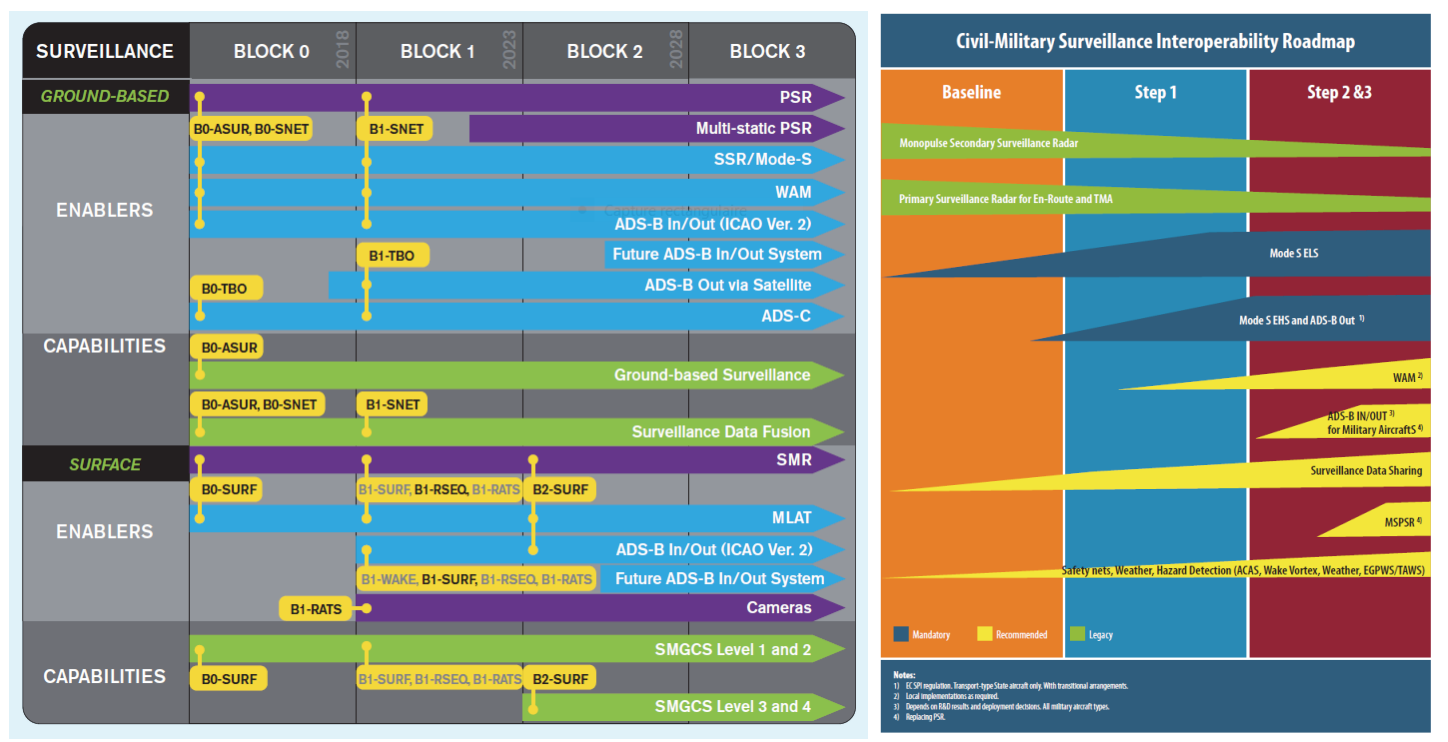

Figure 2. Roadmaps MSPSR \{à gauche OACI (ICAO 2013), à droite Eurocontrol (Eurocontrol 2014)\}

L'horizon est le même (6 à 7 ans) que celui envisagé par les industriels; il semble donc bien que les organismes étatiques considèrent que le marché est maintenant prêt ; l'avenir dira si cette perception est réaliste.

Les travaux qui ont été conduits ont donc bien contribué à inscrire ce nouveau concept de MSPSR dans le paysage de l'ATM du futur, mais paradoxalement, c'est peut-être au sein de l'entreprise que la révolution reste à faire.

En effet, et c'est probablement le bilan le plus intéressant de cet exemple, l'innovation la plus importante concerne maintenant l'organisation de l'entreprise. Comme on l'a vu plus haut, il faut s'intéresser de front à plusieurs aspects; l'innovation technique porte sur de nombreux métiers, qu'il s'agit donc de regarder chacun d'un œil neuf, puis tous ensemble d'un œil neuf. Cela implique donc une innovation organisationnelle, un chantier qui reste à ouvrir.

\subsection{Exemple 3 : Cohabitation entre radars et éoliennes}

Le développement des énergies renouvelables est ralenti par la nécessité de faire cohabiter les nouvelles infrastructures avec les équipements déjà en place. C'est notamment le cas des éoliennes et des radars, les éoliennes constituant des obstacles pouvant perturber le bon fonctionnement des radars, par exemple en créant des détections parasites ou encore en modifiant la propagation des ondes \{le lecteur pourra se reporter à la référence (Moruzzis et Perret 2014) pour une présentation plus complète du sujet\}.

C'est la raison pour laquelle on parle de «cohabitation » entre les éoliennes et les radars, qui sera abordée ici sous l'angle des améliorations qui peuvent être apportées aux radars pour réduire l'impact sur leur propre fonctionnement de la gêne due aux éoliennes, contribuant en cela au développement harmonieux des énergies renouvelables. 


\subsubsection{De quoi s'agit-il ?}

Toutes les nations du monde affirment leur volonté de développer les énergies renouvelables, ce qui se traduit par exemple, en ce qui concerne la production d'électricité par l'éolien en France, par le graphique suivant :

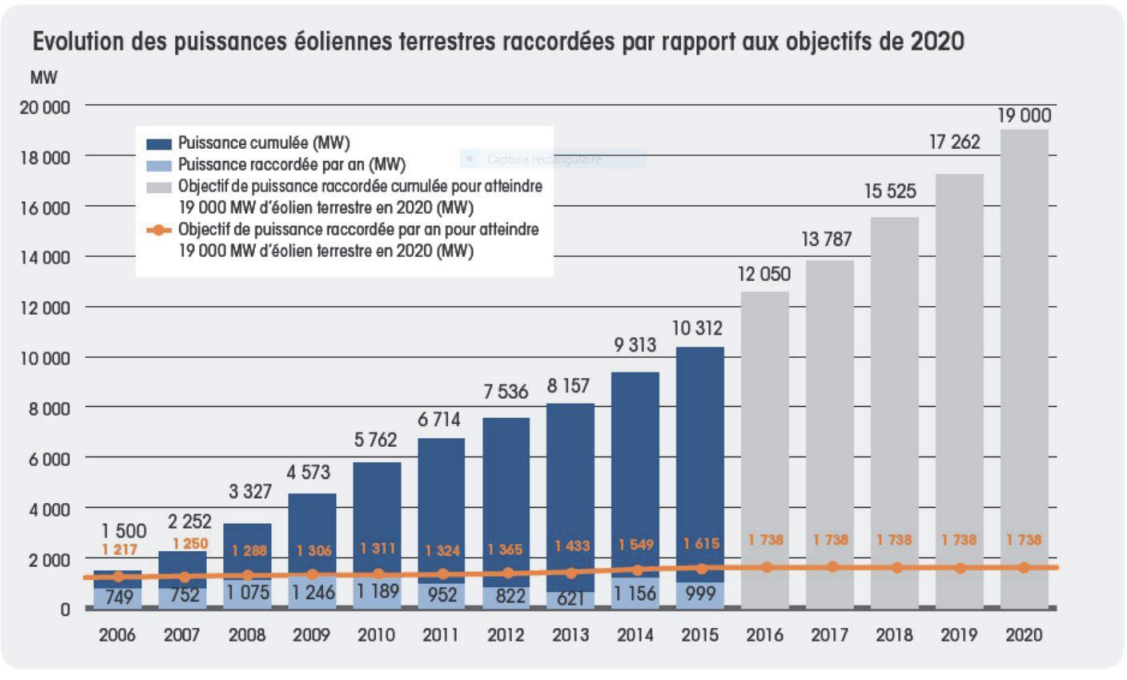

Figure 3. Evolution énergie éolienne en France (source SER)

Le problème est que les projets éoliens sont souvent bloqués à cause des objections, qui se traduisent par des refus de permis de construire, faites par les différents « opérateurs radar » (en France : Défense Nationale, Météo-France, Aviation Civile, Ports et Navigation Maritime). La légitimité de ces objections est souvent mise en cause par les porteurs de projets éoliens, et en l'absence de certitudes, les opérateurs radar se réfugient souvent derrière le principe de précaution. C'est ainsi que de nombreux contentieux se font jour. Ce schéma se retrouve partout dans le monde, avec bien entendu des spécificités liées aux stratégies et particularités locales.

Il s'agit alors de trouver des solutions pour réduire voire supprimer les impacts éoliens. Mais comme on va le voir, cela ne peut se faire qu'en immersion totale dans un environnement complexe mettant en jeu de nombreux acteurs aux stratégies parfois opposées.

\subsubsection{Quels sont les objectifs?}

L'objectif initial de l'auteur et de son entreprise était de comprendre les problèmes posés par les éoliennes aux radars et de chercher des solutions. Bien vite il est apparu à l'auteur que pour atteindre cet objectif il faudrait déployer des efforts importants à des niveaux très variés pour prendre en compte les besoins spécifiques des opérateurs éoliens (notamment en termes de rapidité de déploiement des solutions) et ceux des opérateurs radars (en particulier pour qu'ils aient l'assurance que les solutions proposées leur permettront de maintenir leurs missions opérationnelles).

\subsubsection{Quels travaux ont été réalisés?}

Nous ne parlerons pas ici des activités conduites sur le plan technique ${ }^{4}$, mais plutôt du cadre dans lequel ces travaux se déroulent. Tout d'abord l'environnement externe; il est en effet nécessaire de dialoguer avec les différents acteurs du domaine qui sont principalement :

4. Rentrer dans le détail des travaux entrepris serait en effet fastidieux, mais on peut les résumer en disant que les principales briques permettant de proposer des solutions sont en place \{moyens de calcul des impacts, solution radar comme de nouveaux traitements pour filtrer les échos parasites (Moruzzis, Muller et Ferrier 2010) et que des premières réalisations ont déjà été mises en place\}. 
- les opérateurs éoliens ; il s'agit des entreprises qui développent les projets éoliens. Il peut s'agir de petites structures ou de grands groupes du secteur de l'énergie. Ils sont les demandeurs de solutions et donc les interlocuteurs directs des industriels radar qui peuvent proposer des solutions. Il faut également associer à ce groupe les syndicats de la profession éolienne (nationaux, européens, mondiaux) ainsi que les industriels fabricant les éoliennes ;

- les opérateurs radar (en France : Défense Nationale, Météo-France, Aviation Civile, Ports et Navigation Maritime) ; ils sont les clients finaux (« end-user ») habituels des industriels radar mais ne jouent pas ici ce rôle car ils ne sont pas demandeurs de solution. En revanche c'est avec eux que va se dérouler le dialogue technique et c'est eux qui, in fine, sont décisionnaires en termes d'acceptation ou de rejet d'une solution proposée par le tandem (opérateur éolien + industriel radar);

- les organismes étatiques (en France : DGA, DIRCAM, DGSIC, DGAC, CETMEF, DGPR, ADEME, ANFR, ENAC, ONERA, etc...) qui sont en charge d'élaborer les règlementations, de normaliser les exigences, de piloter les études et superviser les aspects contractuels et d'assurer une expertise technique.

On conçoit facilement que le dialogue puisse être délicat, d'autant plus que les relations entre les intervenants sont inhabituelles, et sortent largement du cadre usuel client-fournisseur.

Il y a notamment au moins trois domaines d'activité de l'entreprise qui se trouvent particulièrement concernés par ce sujet :

- le cadre contractuel («business model ») : comment résoudre le problème du financement, celui de la maintenance de la solution (qui porte typiquement sur plusieurs dizaines d'années), celui des engagements de performance et celui des garanties contractuelles ? Comment gérer la mise en œuvre d'une solution transitoire ? Quel est le juste prix de la solution (en particulier doit-il dépendre de la « taille » du parc éolien) ? Est-il préférable de penser « équipement » ou « service », achat ou location ? Comment traiter correctement le cas où la solution déployée au bénéfice d'un opérateur permet l'implantation ultérieure d'un ou de plusieurs autres parcs éoliens?

- la méthodologie d'engagement des performances : comment qualifier et comment être sûr de maîtriser correctement les impacts ? quels moyens mettre en œuvre pour prédire ces impacts ? comment justifier le besoin - ou l'absence de besoin - d'une solution (et laquelle choisir lorsque plusieurs possibilités se présentent) ? comment quantifier les apports d'une solution pour prendre des engagements de performance?

- la gestion de l'image de l'entreprise : le sujet présente la particularité d'être très visible médiatiquement et politiquement parlant. Il est donc nécessaire de gérer l'image de l'entreprise de manière particulière et ceci d'autant plus que le rôle que peut jouer un industriel radar dans ce domaine n'est pas trivial a priori.

Le dernier point mérite probablement d'être développé un peu plus car c'est un aspect des choses qui est apparu comme le plus inhabituel (et le plus frustrant pour l'auteur). Il s'agit en effet d'un positionnement paradoxal : l'industriel radar est le mieux à même de maîtriser les aspects techniques relatifs d'une part à la compréhension de la gêne occasionnée par les éoliennes et d'autre part aux solutions aptes à y remédier, car il bénéficie notamment d'un accès permanent à la « réalité terrain » (comment fonctionnent les vrais radars dans des vrais environnements). Il est donc le mieux placé pour mettre en œuvre une méthodologie d'analyse et pour proposer des solutions crédibles pour ce qui concerne l'analyse des impacts et les solutions radar. Mais comme il est aussi en mesure d'en tirer un bénéfice financier, il est forcément suspect d'être juge et partie, ce qui vient compliquer sa participation aux yeux des autres partenaires.

Pour tenter de résoudre cette difficulté, un des moyens qui a été promu par l'auteur a consisté à proposer de mettre à disposition des organismes étatiques des outils d'analyse et de prédiction d'impact qui avaient déjà été développés pour les besoins propres de l'entreprise (Moruzzis 2011). L'objectif de la mise à disposition de ces outils était de mettre en place une méthode transparente permettant à toutes les parties de progresser de manière conjointe et cohérente sur la compréhension et la maîtrise des impacts et sur les engagements de performance. A la connaissance de l'auteur, cette position n'a pas encore retenu l'intérêt des services étatiques qui préfèrent, sur ce sujet, s'en tenir pour l'instant à une procédure plus classique de contractualisation avec des organismes considérés comme experts impartiaux. 
Le sujet reste bien sûr d'actualité ; par exemple l'ANFR pilote actuellement une étude (ANFR 2016) concernant l'impact des éoliennes sur les radars pour tenir compte de l'évolution des technologies et des caractéristiques, tant des éoliennes que des radars.

\subsubsection{Quel est le bilan?}

On peut considérer que le sujet est maintenant maîtrisé de manière satisfaisante sur un plan technique, mais que le chemin est encore long pour trouver des solutions appropriées aux autres domaines, et en particulier pour ce qui concerne la méthode permettant de prédire les impacts et la méthodologie de gestion des risques associés à cette prédiction

Il serait donc probablement utile pour la communauté que soient trouvées des solutions aux problèmes non techniques, en particulier pour ce qui concerne les aspects contractuels et méthodologiques. Pour cela on pourrait s'inspirer de travaux initialisés par ailleurs, notamment en Grande-Bretagne qui joue un rôle de pionnier dans ce domaine.

On peut également noter que l'accès aux informations de référence (concernant par exemple les règles à respecter pour l'implantation des parcs éoliens) reste difficile. En France le sujet concerne deux ministères, le Ministère de l'Environnement, de l'Energie et de la Mer (MEEM) d'une part, et le Ministère de la Défense d'autre part, avec la particularité que le MEEM gère à la fois l'aspect éolien et l'aspect radar (pour les radars météo, les radars civils et les radars côtiers). Une conséquence de cette répartition des responsabilités est que la procédure à suivre (MEEM 2015) pour s'assurer de la conformité d'un projet éolien avec les contraintes radar dépend du type de radar, ce qui complexifie les démarches à entreprendre par les développeurs éoliens, et ce qui oblige les industriels radar à s'adapter à ces différentes procédures pour ce qui concerne l'expression des performances par exemple.

La capacité d'un industriel à faire évoluer un paysage aussi vaste reste bien sûr assez limitée (et d'ailleurs ce n'est pas son rôle), mais en continuant à contribuer aux travaux des différentes instances concernées, on peut espérer que ces travaux, notamment ceux conduits sous l'égide de l'ANFR (voir plus haut), viendront simplifier ce paysage et faciliter les procédures. Une telle simplification serait en soit une réelle innovation, et permettrait alors de développer en France une cohabitation harmonieuse et efficace entre les éoliennes et les radars.

\section{Les freins à l'innovation}

Les trois exemples présentés plus haut sont révélateurs de plusieurs mécanismes qui freinent l'innovation. En effet, le premier exemple a montré qu'il est difficile de faire évoluer les pratiques pour passer des études purement techniques aux études fonctionnelles, le deuxième a mis en lumière le besoin pour l'entreprise de remettre en cause son organisation pour bien gérer les ruptures technologiques et le troisième a permis de comprendre que l'entreprise doit apprendre à fonctionner dans un univers complexe pour y trouver sa place et pouvoir y jouer un rôle moteur.

Comme ces mécanismes ne sont que le résultat de quelques « sondes » constituées par les trois exemples, il a semblé utile à l'auteur de voir si on pouvait leur trouver un certain caractère de généralité, en tentant de faire un panorama plus général des mécanismes qui viennent entraver l'innovation. Pour cela l'auteur a tenté d'identifier et de classer de tels mécanismes selon trois catégories :

- les freins généraux ;

- les freins spécifiques aux entreprises industrielles ;

- les freins spécifiques à l'environnement des entreprises industrielles. 


\subsection{Les freins généraux}

Les entreprises industrielles n'échappent pas à des blocages classiques qui affectent toute activité humaine, et dont certains (sans prétendre bien sûr à l'exhaustivité en la matière) sont détaillés dans ce qui suit.

\subsubsection{La peur d'innover}

La nature humaine est ambivalente ; elle est attirée par les choses nouvelles mais elle en a aussi peur. En se concentrant plus spécifiquement sur nos réticences face aux choses nouvelles, l'auteur a identifié trois peurs qui traduisent nos difficultés à entreprendre :

\subsubsection{La peur de faire des choses trop « difficiles »}

Le plus simple pour en parler est d'évoquer deux exemples :

- dans une de ses lettres à Lucilius (Baillard 1914), Sénèque explique que « Ce n’est point parce qu’il est difficile que nous n'osons pas ; c'est parce que nous n'osons pas, qu'il est difficile. »;

- bien plus tard, dans son discours de 1962 (Kennedy 1962) (extrait en Annexe), John Fitzgerald Kennedy a proposé au peuple américain de se lancer dans l'aventure du programme Apollo non pas parce que c'était facile, mais bien parce que c'était difficile ${ }^{5}$.

La question qui nous est posée est bien celle de l'attirance de l'esprit humain pour les choses difficiles, alors que la société actuelle et notamment l'officialisation du «principe de précaution » nous pousse plutôt à la prudence et à la minimalisation des risques. Le lien avec l'innovation est direct puisqu'innover implique d'entreprendre.

\subsubsection{La tentation d'innover sans prendre de risque}

On aborde ici un mécanisme bien expliqué par certains auteurs (Koetzier et Alon 2013) qui consiste à penser qu'en limitant le périmètre de l'innovation on va réduire les risques. C'est un réflexe assez naturel et qui semble issu du principe de conservation, mais, en pensant trouver une parade à la peur de faire des choses sortant des sentiers battus, il condamne l'entreprise à une sorte de suivisme et lui enlève justement le goût du risque. Le désir d'oser est constitutif de l'action d'entreprendre : une " organisation » qui n'entreprend pas n'est pas une entreprise mais une administration.

C'est peut-être une manière de faire fonctionner l'entreprise à court ou moyen terme, mais cela met en danger sa capacité à évoluer et à penser « plus loin » (ou à penser tout court). Dans un marché ouvert à la concurrence, si cela devient un principe de management de l'entreprise, elle est condamnée à plus ou moins long terme.

\subsubsection{La peur d'échouer}

Il s'agit là d'un mécanisme culturel, que l'on retrouve particulièrement exacerbé dans la culture et l'éducation françaises.

R. Sutton parle très bien de cette peur d'échouer et propose des solutions pour lutter contre ses effets dans les entreprises (Sutton 2001); il explique par exemple que très souvent on récompense le succès et on punit l'échec. Cela a pour conséquence désastreuse de freiner les initiatives, car on n'aime en général pas courir le risque d'être puni.

Pour remédier à cela, il propose une approche qui nous semble iconoclaste, mais qui a le mérite de nous faire réfléchir de manière inhabituelle ; il propose en effet :

- de récompenser le succès ;

- de récompenser l'échec de la même manière que le succès ;

- de punir l'inaction. 
Sans aller jusqu'à cet extrême, l'entreprise peut au moins s'inspirer de certaines approches comme la dépénalisation des erreurs non intentionnelles qui, lorsqu'elle est clairement affichée, est un encouragement à entreprendre ; cela présente aussi l'avantage de rendre beaucoup plus efficace la gestion et la prise en charge des erreurs (voir notamment (Morel 2012)).

\subsubsection{La difficulté de passer de l'invention à l'innovation}

En se concentrant sur les innovations dans les entreprises industrielles, l'auteur a pu observer que l'invention est la plupart du temps du ressort de l'individu alors que l'innovation est une affaire de groupe (car il s'agit alors de passer à une phase de développement qui concerne différents services ou métiers). Pour passer de l'un à l'autre il faut que l'idée soit transmise, c'est-à-dire qu'elle soit à la fois bien expliquée (par l'inventeur) et bien comprise (par le groupe qui se chargera du développement).

Selon l'expérience de l'auteur, le mécanisme consistant à décider du lancement de la phase de développement (analyse du projet d'innovation) à partir de la phase initiale (invention) est maintenant bien passé dans la culture d'entreprise.

Mais cela ne suffit pas, car cela suppose ensuite un effort de communication de la part de tous les intervenants. Mais ce qui est souvent sous-estimé c'est le fait qu'une idée ne peut pas se développer si les autres ne se l'approprient pas, s'ils ne se sentent pas impliqués ou s'ils n'y adhèrent pas. Il ne faut pas négliger non plus le temps qui est nécessaire à cette phase d'incubation.

Une meilleure compréhension de ces mécanismes et un encouragement à franchir ce passage de l'invention à l'innovation permettrait d'éviter que tant de projets restent « dans les cartons » (une autre spécificité bien française).

\subsection{Les freins spécifiques aux entreprises industrielles}

Il s'agit ici de considérations plus spécifiques au fonctionnement des entreprises et à leur organisation interne.

\subsubsection{Le complexe scientifico-moderniste et autres syndromes}

Pour l'auteur, il existe un complexe \{appelons-le « scientifico-moderniste »; mais on pourrait aussi le nommer NIB (New Is Beautiful)\}, qui consiste, de la part de l'entreprise, à considérer que la dernière «nouveauté » issue d'un laboratoire de recherche, présentée dans une conférence ou dans un article scientifique est forcément intéressante pour elle. Promouvoir un tel sujet permet d'attirer l'attention (et parfois des financements) sur des thèmes toujours présentés comme «novateurs », mais cela a souvent aussi pour conséquence de ralentir voire d'empêcher la mise en place de solutions industrielles.

Ce syndrome s'accompagne en général d'un langage sophistiqué, permettant de renommer des choses existantes mais de manière plus « savante ». Cette manière de faire est problématique en particulier pour les plus jeunes générations car elle sème la confusion dans les esprits en empêchant de voir que, sous des noms différents se cache le même procédé. Dans le monde du radar, on peut citer par exemple quelques termes actuellement en vogue :

- « Micro-Doppler » (Chen, et al. 2003), pour parler de la signature Doppler ;

- «MIMO Radar » (Chapursky, et al. 2009) ou encore « Radar tomographique », qui désignent en fait un radar multistatique ;

- « Radar holographique » (Aveillant 2016), un terme plus élégant que « radar à FFC» (Formation de Faisceau par le Calcul). 
D’autres syndromes plus connus peuvent avoir des conséquences similaires :

- le NIH (Not Invented Here), qui fait que l'on ignore volontairement les travaux déjà réalisés à l'extérieur - une maladie qui s'apparente à réinventer la roue et qui touche principalement la population des « inventeurs »-;

- le virus du manque de confiance - une autre forme d'affection qui touche, elle, plutôt les cadres de l'entreprise, et que l'on pourrait nommer OIB (Outdoor Is Better) - qui fait qu'on pense que l'idée du concurrent est forcément meilleure que la sienne.

Une meilleure formation des cadres de l'entreprise à ces mécanismes ainsi qu'une meilleure connaissance de ses propres forces et faiblesses permettrait évidemment d'atténuer les effets de ces complexes et syndromes.

\subsubsection{Faiblesse d'analyse/de compréhension du monde extérieur}

Face aux évolutions rapides du monde environnant l'entreprise, il est indispensable qu'elle s'organise pour bien le comprendre et pour être capable de réagir au moment opportun.

Malheureusement, les fonctions de type « marketing » et « stratégie » sont souvent les parents pauvres de nos entreprises, et/ou leur apport n'est pas suffisamment intégré dans les mécanismes de pilotage de l'innovation.

Renforcer le rôle de ces fonctions et développer une mentalité d'ouverture et de curiosité pour la manière de travailler de nos voisins (notamment Européens et surtout Anglo-saxons) serait déjà une bonne avancée.

\subsubsection{Une organisation interne mal adaptée à l'innovation}

L'organisation des entreprises est souvent cloisonnée (on peut d'ailleurs souvent parler de « silos »), alors que l'innovation devrait la concerner dans son ensemble. Ce sujet est bien traité dans certains ouvrages auxquels le lecteur pourra se référer pour approfondir ce point (voir par exemple (Sutton 2001) et (Koetzier et Alon 2013)).

La nécessité d'une bonne synergie entre stratégie et innovation est en particulier assez évidente, comme on peut le percevoir à travers l'exemple présenté plus haut et concernant les éoliennes.

Un autre défaut de l'organisation réside aussi souvent dans le «casting » des responsables en charge du management de l'innovation : quelle que soit la structure qui est mise en place pour cela, il est tentant de recruter ces ingénieurs dans la population des inventeurs. Si cela présente un intérêt évident lié à l'expérience de l'individu, il ne faut pas ignorer que cela peut comporter un risque qu'il considère (plus ou moins consciemment) cette position comme un moyen de faire aboutir ses propres idées, ce qui constituerait un détournement assez grave de la fonction. Un profil mieux adapté serait sans doute à trouver dans la population des responsables de programme (ou chefs d'affaire) ayant déjà une bonne expérience des différents métiers de l'entreprise et de sa stratégie globale.

Le choix d'un responsable de l'innovation (ou d'un directeur technique) est particulièrement délicat puisqu'on attend de lui, en plus d'une bonne connaissance du domaine technique, qu'il sache encourager le développement des bonnes idées de manière réfléchie (c'est-à-dire en s'appuyant sur une bonne connaissance des besoins et des capacités de l'entreprise), constructive (notamment en sollicitant l'avis des experts) et transparente (dans le but de susciter l'adhésion, par exemple en expliquant les choix et les stratégies retenues). Ayant également un rôle moteur, il doit avoir une bonne capacité de conviction et d'entraînement, tout en sachant faire respecter ses décisions. Il s'agit donc bien d'un casting difficile, qui nécessite une grande capacité de jugement de la part de la Direction de l'entreprise qui le choisit.

Dans un autre domaine touchant l'organisation, l'entreprise devrait s'inquiéter du risque croissant de volatilité de son capital intellectuel. En effet le développement des nouvelles techniques numériques 
facilite l'accès individuel à un grand volume d'informations, et la production d'un grand nombre de données et résultats, mais leur capitalisation et leur archivage nécessite une stratégie raisonnée de gestion des connaissances. Outre une perte d'efficacité globale, la négligence en la matière peut aussi constituer un frein à l'innovation dans la mesure où l'entreprise peut oublier des travaux qu'elle a déjà réalisé (et donc les refaire) ou ne plus pouvoir accéder à certaines informations précieuses.

Deux anecdotes illustrent cette "volatilité »; elles concernent un programme datant du milieu des années 90, le premier pour lequel une documentation numérique complète avait été fournie au client :

- dix ans plus tard il était devenu impossible d'y accéder car le support physique (disque souple) et le logiciel de traitement de texte étaient devenus obsolètes ;

- vingt ans plus tard, on a donné le même nom à un nouveau programme concernant pourtant la même famille de produit. Le client et le fournisseur avaient visiblement oublié le précédent (ou plutôt en ignoraient complétement l'existence).

\subsection{Les freins liés à l'environnement des entreprises industrielles}

L'entreprise fait partie d'un milieu avec lequel elle interagit; il est donc important d'essayer de comprendre quels peuvent être les freins liés à cette interaction.

\subsubsection{Etudes amont et recherche appliquée}

«Etudes amont» désigne souvent des travaux préliminaires ou exploratoires, mais qui auront une suite plus « aval », c'est-à-dire appliquées à un développement. Cela découle d'une vision logique de déploiement de travaux destinés à atteindre un objectif précis (la vente d'un nouveau produit ou l'amélioration d'un produit existant). Le lecteur peut donc imaginer que ce cheminement résulte d'une stratégie destinée à atteindre cet objectif, même si le chemin pour y arriver n'est pas encore bien connu, ce qui nécessite, justement des « étude amont» pour le défricher.

C'est en cela que les études amont se différencient de la « recherche », qui ne nécessite pas forcément l'existence d'un objectif de développement. Et c'est bien l'existence ou non de cet objectif de développement qui doit normalement différencier les travaux conduits par les industriels d'une part et par les organismes de recherche d'autre part.

Selon l'auteur, cette distinction reste en France trop virtuelle, par un défaut de pilotage des travaux d'étude par les organismes en charge d'organiser justement la coordination entre les organismes de recherches et l'industrie (France Stratégie 2016) et (Berger, Dorman et Starbuck 2016) et par une confusion des rôles qui nuit à l'efficacité de l'ensemble.

Il n'y a en effet pas de vision réellement partagée entre les différents acteurs, et pas de coordination efficace des travaux à réaliser par les industriels et par les organismes de recherche. Il est alors possible de constater la formation de situations paradoxales dans lesquelles :

- Par manque de vision stratégique suffisamment mature, affinée et partagée au sein de leur entreprise, des industriels réalisent des travaux prospectifs (par exemple autofinancés) sans réel horizon d'application. Cela peut être notamment une conséquence du phénomène des « donjons » (ou chapelles ou silos) qui empêche l'élaboration d'un cap raisonné ;

- Des industriels doivent faire (la plupart du temps dans l'urgence) des études nécessaires au développement d'un produit parce que ces travaux n'ont pas été réalisés par les organismes de recherche dont c'est normalement la mission ;

- Pour différentes raisons (par exemple par manque de pilotage du donneur d'ordres et/ou par manque de connaissance de l'état de l'art et/ou par manque d'ambition technique, et/ou tout simplement par paresse intellectuelle - pourquoi faire des choses difficiles? -), des organismes de recherche travaillent sur des sujets qui ont déjà été résolus par des industriels, ou qui sont considérés ad vitam aeternam comme « prospectifs ». Une pincée de complexe scientifico-moderniste (voir plus haut) étant alors suffisante pour refaire les mêmes études tous les dix ans. 
Et pourtant ce n'est pas faute de structures et d'organismes en charge de piloter les travaux. Par exemple pour la DGA l'innovation est structurée en deux grands axes ${ }^{6}$ :

- «Recherche et Innovation Scientifique », dont les objectifs sont définis dans le document «POS» (Politique et Objectifs Scientifiques) qui « oriente l'effort d'investissement consenti par la Défense sur la partie basse du spectre de maturité des technologies »;

- «Etudes amont», définies comme des «recherches et études appliquées, de nature technique, entreprises en préparation du lancement des programmes d'armement ».

Mais il n'y a malheureusement pas de coordination réellement efficace entre les industriels et les organismes de recherche. On s'attendrait par exemple à ce que d'une bonne coordination ressorte une analyse des travaux à mener à court/moyen ou long terme, et à ce que l'Etat fixe aux organismes de recherche des objectifs beaucoup plus ambitieux sur le long terme (voir Annexe « POS »).

Cette question de la coordination entre les organismes de recherches et l'industrie est d'ailleurs au cœur du débat actuel sur l'efficacité de l'innovation en France. Des rapports officiels récents pointent le foisonnement des dispositifs nationaux de soutien à l'innovation - 62 ont été recensés en 2015 - voir (France Stratégie 2016) et leur inefficacité.

A tel point qu'au schéma actuel qui est censé résoudre les difficultés à interfacer les organismes de recherche avec les industries par la multiplication de mécanismes de transfert, certains experts (Berger, Dorman et Starbuck 2016) recommandent un schéma radicalement différent :
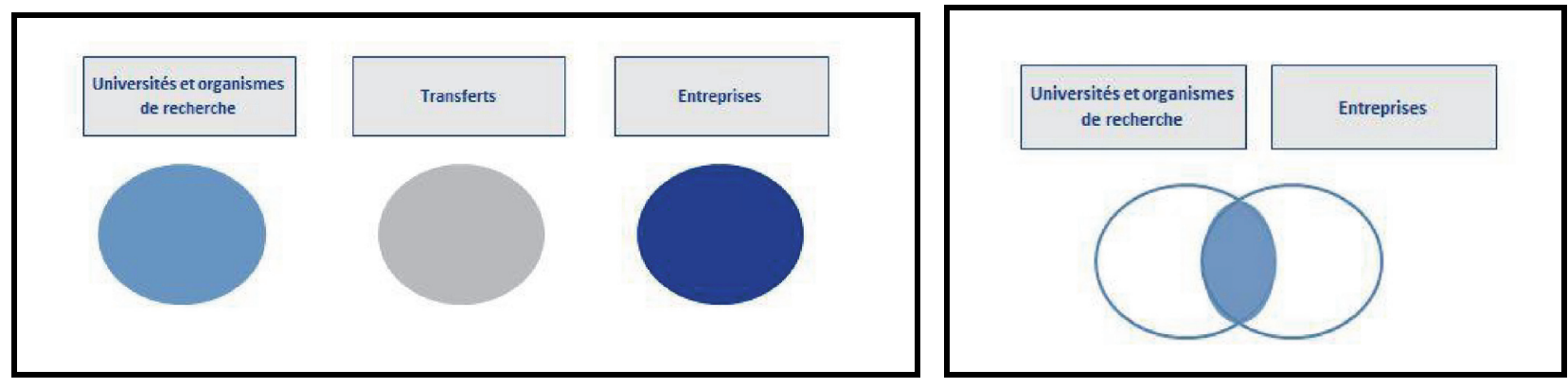

Figure 4. Interface Recherche-Industrie

(à gauche : schéma actuel, à droite schéma recommandé)

\subsubsection{Manque de synergie avec le monde extérieur}

On a commencé à aborder le sujet en évoquant les problèmes de faiblesse de l'analyse et de la compréhension du marché (3.2.2), mais cela peut être complété en mentionnant les aspects suivants :

- S'agissant d'innovation, il est naturel d'ignorer plusieurs éléments du problème et de ne pas être capable de trouver seul les bonnes réponses. Un défaut courant consiste à imaginer que l'intéressé va résoudre les difficultés par ses propres moyens; c'est non seulement présomptueux mais très souvent contre-productif. Si on reprend les trois cas présentés plus haut, l'auteur a par exemple été confronté au problème d'arriver à énoncer clairement des critères de performance; il s'est avéré que dans tous les cas ce n'est qu'en ouvrant la discussion avec des intervenants extérieurs (les utilisateurs finaux de préférence) qu'il a été possible d'avancer et de proposer des réponses efficaces ;

- C'est le même problème lorsqu'il s'agit d'anticiper une transition; avec qui d'autre que les utilisateurs peut-on arriver à imaginer quel sera le bon moment ? 
- Là où la situation peut se compliquer, c'est lorsqu'il devient indispensable de fédérer une communauté plus large. Le cas des éoliennes (2.3) est un bon exemple : pour avancer dans la mise en place pragmatique de solutions, on peut s'inspirer par exemple de ce qui a été fait en Grande-Bretagne concernant la mise en place d'un « Aviation Plan » ${ }^{7}$. Cela nécessite de pouvoir innover en termes d'organisation, mais on peut rapidement se heurter à un manque d'intérêt ou de volonté de la part des organismes dont c'est normalement la responsabilité ; que peut alors faire un industriel à part suggérer la mise en œuvre d'une méthode de ce type ?

- Il existe de nombreux groupes de travail touchant à l'innovation et à différents niveaux (national, européen, OTAN, etc...). La participation à ces groupes est très souvent réservée aux représentants des organismes étatiques, mais les industriels ne bénéficient que marginalement des retombées des travaux de ces groupes. Il est vrai qu'une certaine ouverture commence à se faire, et c'est un bon signe, mais la frilosité continue à régner malgré tout, surtout en France où le régime de protection des informations est totalement asymétrique avec le partage léonin du « droit à en connaître » par principe réservé quasiment exclusivement à la puissance publique. Il n'est pas rare, par exemple que des industriels n'interviennent que sur « invitation » pour faire part de leur expérience ;

- Enfin, et c'est surtout vrai quand il s'agit d'aborder la maitrise des performances et leurs méthodologies de mesure, il devient urgent de changer d'approche, et de mettre enfin en commun les moyens et outils de calcul qui permettront à tous les intervenants d'appréhender le sujet et de gagner en confiance réciproque. Ce type d'approche commence à exister, heureusement, mais il s'agit encore d'initiatives isolées (notamment dans le domaine de l'aviation civile) où certains clients demandent maintenant non seulement des équipements mais aussi les moyens de calcul permettant de prédire leurs performances dans des environnements et des conditions variés.

\section{Que faire pour améliorer la situation?}

Ce chapitre est consacré aux pistes qui pourraient aider les entreprises à mieux innover. L'auteur, en tant qu'observateur attentif, y suggère quelques pistes, mais laisse bien sûr aux spécialistes du management de l'innovation le soin de les formaliser et de leur trouver une traduction pratique.

L'enseignement principal que l'auteur a tiré de sa propre expérience est que l'innovation ne peut pas reposer sur quelques individus (quelle que soit leur expertise et leur volonté); elle doit être prise au sérieux et intégrée dans l'entreprise comme un de ses ressorts vitaux. Pour cela, il faut beaucoup mieux former et préparer ses cadres à la conduite du changement, à la complexité, à l'invention, à l'innovation et à la propriété intellectuelle.

L'entreprise devrait aussi s'interroger sur son niveau d'investissement dans les études de marché pour comprendre et analyser au mieux les tendances et évolutions. C'est vrai également pour ce qui concerne les solutions innovantes développées par nos voisins, en particulier Européens (mais pas seulement). L'entreprise devrait également questionner sa structure pour s'assurer qu'elle permet de bien gérer l'innovation multi-domaine.

Pour lutter contre les différents syndromes et complexes, l'entreprise pourrait améliorer l'efficacité de la veille technique et développer une meilleure analyse critique de maturité et d'adéquation avec ses besoins, ce qui va de pair avec une meilleure diffusion interne de ses propres travaux et connaissances. 
Un aspect de la diffusion de la connaissance concerne la stratégie. Pour bien cibler les travaux et les orienter, il faudrait que la stratégie soit d'une part bien diffusée aux personnes concernées, et que d'autre part elle s'alimente en permanence des avancées en la matière (en interne et en externe). Une bonne synergie entre la stratégie, le marketing et l'innovation est particulièrement cruciale.

A plusieurs reprises (par exemple au moment de la préparation des offres d'études innovantes) l'auteur a pu constater que l'ampleur des activités et surtout la complexité de leur environnement étaient sousestimées. Une analyse plus approfondie en amont permettrait de corriger ce défaut.

Innover en entreprise c'est aussi structurer l'accès à ses propres connaissances. Il faudrait par exemple s'attacher à mieux définir quelle est la « bonne » information dont l'entreprise aura besoin, comment la mémoriser pour que d'autres puissent y accéder, par exemple dans 10 ou 20 ans, et moderniser les outils associés. L'auteur n'est pas un spécialiste de ces questions, mais il peut constater tous les jours la distance qui sépare les outils mis à disposition par les entreprises et ceux, beaucoup plus performants, auxquels il accède dans sa vie privée, par exemple en ce qui concerne les moteurs de recherche

Dans ses relations internes mais surtout avec l'extérieur, il ne faut pas négliger l'importance de la pédagogie des choses nouvelles car il n'est jamais évident que les autres perçoivent l'intérêt d'un nouveau concept. Et dans tous les cas il faut prendre conscience du temps nécessaire à ce qu'une nouvelle idée diffuse suffisamment pour que les autres puissent se l'approprier.

Par ailleurs, l'entreprise devrait clarifier ses relations avec ses donneurs d'ordre (notamment étatiques), par exemple en affirmant plus clairement sa stratégie et en contribuant de manière plus active aux instances de coordination et de réflexion, ce qui constitue probablement le moyen le plus efficace, à l'heure actuelle, pour faire évoluer l'organisation de l'innovation au plan national vers plus d'efficacité.

Enfin, il faut encourager les synergies - et pas seulement se contenter de mettre en place des structures de façade comme c'est trop souvent le cas - entre les différents acteurs (non, ce n'est pas une banalité, et cela demande même une grande pugnacité !), et en particulier décloisonner les relations entre industriels, responsables étatiques, organismes de recherche et utilisateurs finaux, et que tous apprennent à apprendre les « écosystèmes » que vont constituer leurs futurs environnements.

\section{Conclusion}

Pour Robert Bozza, « l'innovation c'est la rébellion » (N'Kaoua 2013).

Le film « La solitude du coureur de fond » raconte que pour aider à la réinsertion d'un jeune pensionnaire rebelle (Colin Smith), le directeur d'un centre de rééducation ${ }^{8}$ encourage son aptitude à la course de fond pour qu'il remporte une épreuve de cross-country. Mais au moment où Colin est sur le point de gagner la course, sa soif de liberté lui fait faire un autre choix.

La fin de l'histoire n'aurait-elle pas été plus heureuse pour tout le monde, si le directeur avait encouragé Colin à pratiquer un sport d'équipe ?

L'innovation doit bien sûr rester une rébellion, mais son rendement restera faible tant qu'il ne s'agira que d'aventures individuelles; pour améliorer l'efficacité de l'entreprise, son management serait bien inspiré de trouver le moyen de l'organiser pour innover collectivement.

Et donc, au final, n'est-ce pas dans le domaine de l'organisation que l'entreprise a le plus besoin d'innovations?

8. L'auteur tient tout de même à préciser qu'il n'assimile pas les entreprises industrielles à des centres de rééducation ! 


\section{Acronymes}

ADEME

ADN

ANFR

ATC

ATM

CDR

CEA

CIDEF

CNES

CNRS

DGA

DGAC

DNA

ENSTA

FFC

GDR

GIFAS

ICAO

INRIA

INSA

INSERM

IPSIS

ISAE

ISIS

IVVQ

MEEM

MIMO

MSPSR

NCTR

NIB

NIH

$\mathrm{OACI}$

OFCOM

OIB

ONERA

OTAN

$\mathrm{PhD}$

PME

POS

PSR

RCS

SAT

SDR

SER

SER

SESAR

TU
Agence De l'Environnement et de la Maîtrise de l'Energie

Acide Désoxyribonucléique

Agence Nationale des Fréquences

Air Traffic Control

Air Traffic Management

Critical Design Review

Commissariat à l'Energie Atomique

Conseil des Industries de Défense Françaises

Centre National d'Etudes Spatiales

Centre National de la Recherche Scientifique

Direction Générale de l'Armement

Direction Générale de l'Aviation Civile

Deoxyribonucleic Acid

Ecole Nationale Supérieure des Techniques de l'Armement

Formation de Faisceau par le Calcul

Groupement De Recherche

Groupement des Industries Françaises Aéronautiques et Spatiales

International Civil Aviation Organization

Institut National de la Recherche en Informatique et en Automatique

Institut National des Sciences Appliquées

Institut National de la Santé Et de la Recherche Médicale

Ingénierie pour Signaux et Systèmes

Institut Supérieur de l'Aéronautique et de l'Espace

Information Signal Image et vision

Integration Verification Validation Qualification

Ministère de l'Environnement de l'Energie et de la Mer

Multiple Input Multiple Output

Multi Static Primary Surveillance Radar

Non Cooperative Target Recognition

New Is Beautiful

Not Invented Here

Organisation de l'Aviation Civile Internationale

The Federal Office of Communications

Outdoor Is Better

Office National d'Etudes et de Recherches Aérospatiales

Organisation du Traité de l'Atlantique Nord

Philosophiæ doctor

Petites et Moyennes Entreprises

Politique et Objectifs Scientifiques

Primary Surveillance Radar

Radar Cross Section

Site Acceptance Test

System Design Review

Surface Equivalente Radar

Syndicat des Energies Renouvelables

Single European Sky ATM Research

Technical University 


\section{Bibliographie}

ANFR. Etudes en vue. http://www.anfr.fr/publications/études-en-vue/\#menu2, 2016.

AveILlant. Holographic Radar. http://www.aveillant.com/, 2016.

Baillard J., Oeuvres complétes de Sénèque le Philosophe, Traduction nouvelle avec une notice sur la vie et les écrits de l'auteur et des notes, Tome second. Librairie Hachette et Cie (gallinica.bnf.fr / Bibliothèque nationale de France), 1914.

Berger S., Dorman R., Starbuck H., " Reforms in the French Industrial Ecosystem, Rapport à Monsieur le Secrétaire d'Etat à l'Enseignement Supérieur et à la Recherche et à Monsieur le Ministre de l'Economie, de l'Industrie et du Numérique », 2016.

Billaud P., Colin N., Desodt G., Moruzzis M., Landeghem M., Radars de Surface - Radars Civils et Radars Côtiers Techniques de l'Ingénieur (TE 6678), 2014.

Boulay T., Gac N., Mohammad-Djafari A., Lagoutte J., « Algorithmes de Reconnaissance Non Cooperative de Cibles et Implémentation sur GPU », GRETSI,. Bordeaux, France, 2011.

ChaPURSKY V.V., IVASHOV S.I., VASILIEV I.A., ZhuraVleV A.V., « Short Range Radar with MIMO Antenna System and Multifrequency Sounding Signal », PIERS Online 5, n 8 (2009).

CHEN V.C., Li F., Ho S.S., WeshleR H., « Analysis of micro-Doppler signatures », IEE Proceding on Radar, Sonar \& Navigation 150, $\mathrm{n}^{\circ} 4$ (2003).

DARRICAU J., Physique et Théorie du Radar, $4^{\circ}$ Edition. http://radars-darricau.fr, 2015.

Delhote C., MorUZzis M., « MILORD: A Technical Demonstrator for Long Range Radar Identification » Non-Cooperative Air Target Identification Using Radar. Mannheim, Germany, 1998.

DOREY J., BLANChARD Y., Christophe F., «Le projet RIAS, une approche nouvelle du Radar de surveillance aérienne », Colloque International sur le radar. Versailles, 1984.

ETSI. « Work Program Report» https://portal.etsi.org/webapp/WorkProgram/Report_WorkItem.asp?WKI_ID=47108. 2016.

EUROCONTROL, Roadmap on Enhanced Civil-Military CNS Interoperability and Technology Convergence, Edition 2.0. Eurocontrol 13/10/17-07, 2014, 2014.

EUROCONTROL, Specification for ATM Surveillance System Performance (Volume I), Edition 1.1. Eurocontrol-SPEC-147, ISBN : 978-2-87497-022-1, 2015.

FRANCE STRATEGIE. «Quinze ans de politique d'innovation en France, Rapport de la Commission nationale d'évaluation des politiques d'innovation », 2016.

ICAO. « 2013-2028 Global Air Navigation Plan », ICAO Doc 9750-AN/963, Fourth Edition, 2013.

KENNEDY J.F., Discours du 12 Septembre 1962 (1962).

Koetzier W., Alon A., Why "Low Risk" Innovation is Costly. Accenture, 2013.

MEEM. « Arrêté du 26 Août 2011 (modifié le 6 Novembre 2014 et complété par la décision du 20 Novembre 2015) relatif aux installations de production d'électricité utilisant l'énergie mécanique du vent au sein d'une installation soumise à autorisation... », 2015.

MOREL C., Les décisions absurdes (Tome 2) - Comment les éviter. Gallimard, 2012.

MORUZZIS M., « Alternative Detection Technique to Supplement PSR Coverage (ADT) Final Report », Eurocontrol, 2007.

MORUZZIS M., « Innovation radar et développement éolien », Conférence du bureau de coordination franco-allemand sur les énergies renouvelables. Paris, 2011.

MORUZZIS M., MUlLer D., FerRIER J.M., Système de surveillance multistatique optimisé. Brevet EP 2251706. 2010.

MORUZZIS M., PERRET F., « Mitigation of wind farms impacts, methodology and solutions », IEEE 2014 International Radar Conference,. Lille, France, 2014.

MoruzZis M., COLIN N., « Radar Target Recognition by Fuzzy Logic », IEEE AES Magazine 13, n 7 (1998b).

MoruZzis M., BeAUQUet G., CAMPOY F., Procédé pour filtrer les échos radars produits par les éoliennes. Brevet WO 2010069886. 2010. 
Moruzzis M., Guillerot J.C., LeSTRAde C., «Analysis of experimental data for NCTR target modelling », NATO RTO Symposium on "Non-Cooperative Air Target Identification Using Radar". Mannheim, Germany, 1998a.

MoruZzis M., SAUlais P., Hock TAT TAN., Cherng HuEI TONG., « Automatic Target Classification for Naval Radar », Radar 2004 International Conference on Radar Systems. Toulouse, 2004.

N'KaOUA L., « Robert Bozza, un passionné d'innovation chez France Cleantech », Les Echos, 19 mars 2013.

SESAR. « European ATM Master Plan, Executive View, Edition 2015 », SESAR Joint Undertaking, 2015. —. «SESAR Deliverables on Surveillance rationalisation and the Evolution of the Surveillance Infrastructure », http://www.eurocontrol.

int/publications/sesar-wp-150401-final-deliverables. 2016.

SutTon R., 11.5 idées décalées pour innover. Pearson-Village Mondial, 2001.

TAIT P., Introduction to Radar Target Recognition. The Institution of Engineering and Technology, 2005.

THALES. Projet de thèse: Développement d'algorithmes innovants d'apprentissage et de reconnaissance de cibles noncooperatives à partir de signatures Radar micro-Doppler. jobs.thalesgroup.co.uk, 2016.

HEIDEN R., Aircraft Recognition with Radar Range Profiles. Universiteit van Amsterdam, 1998.

WILLIS N.J., GRIFFITHS H., Advances in Bistatic Radar. Scitech Publishing, 2007.

\section{Annexes/ Citations}

A la lecture du POS (Edition 2010), on peut être surpris par le niveau de détail technique avec lequel étaient fixées les actions prioritaires 2011-2012 du domaine 3 (« Ondes acoustiques et radioélectriques ») concernant la modélisation et la simulation électromagnétique : "Les recherches seront orientées sur la stabilisation en fréquence des méthodes multi-pôles rapides multi-niveaux (MLFMM), sur des résolutions algébriques de la méthode des moments, sur le développement de méthodes asymptotiques (optique physique itérative, sommation de faisceaux gaussiens) et analytiques ». Un tel niveau de précision suppose un suivi extrêmement spécialisé et assidu des travaux, ce qui semble disproportionné par rapport aux moyens mis en place. On s'attendrait plutôt à une définition des orientations en termes d'applications ou de finalité (pour quoi faire ? quelle fonction réaliser ? à quel horizon ?) plutôt qu'en termes scientifiques (comment faire ? quelle méthode utiliser ?). Et ceci d'autant plus que la diversité des intervenants sur ce sujet particulier est loin d'être négligeable. A titre d'illustration, une recherche faite sur Google (en 2016) avec le mot clef « simulation radar » et limitée à la France renvoie vers les entités suivantes : Airbus, Alyotech, CEA, CELAR, CNES, DGAC, ENSTA, INRIA, INSA Lyon, IPSIS, Météo-France, Nexio Group, OKTAL SE, ONERA, SOGITEC, Thales, Université de Bordeaux, Université de Limoges, Université de Rennes, Université de Toulouse, Université d'Evry, Université Européenne de Bretagne. On imagine facilement que la coordination d'un tel domaine demande une vision très stratégique et est difficilement compatible avec la définition de sujets techniques aussi pointus. (POS (Politique et Objectifs Scientifiques, 2010))

"We choose to go to the Moon. We choose to go to the Moon in this decade and do the other things, not because they are easy but because they are hard, because that goal will serve to organize and measure the best of our energies and skills, because that challenge is one that we are willing to accept, one we are unwilling to postpone, and one which we intend to win, and the others, too. ». (Kennedy 1962)

«Innovation is not working out the way many companies expected. Despite increasing commitment, funding and organizational accountability, many companies are disappointed by the returns they are deriving from their investments. Correspondingly, they are scaling back expectations. Instead of the disruptive products, services, and business models that were anticipated several years ago, many initiatives have become considerably more limited in scope. Rather than offering "the next big thing", innovations coming to market today are more typically line extensions. This cautious approach to innovation is understandable, given the relatively disappointing results. At the same time, however, it is a potentially perilous strategy. Enterprises that are able to successfully innovate at a breakthrough 
level can increase the likelihood that they will dominate and prosper in new markets that they create. Enterprises that restrict themselves to incremental innovation, on the other hand, risk unknowingly entering a vicious cycle in which they lag ever farther behind ». (Koetzier et Alon 2013)

- « Idea \#1: Hire people who are "slow learners"

- Idea \#1.5: Hire people who you dislike or feel uncomfortable about

- Idea \#2: Hire people you probably don't even need yet

- Idea \#3: Use job interviews to get ideas, not to screen candidates

- Idea \#4: Encourage people to ignore and defy their peers and bosses

- Idea \#5: Identify your "happy" people and get them fighting

- Idea \#6: Reward success and failure equally generously but punish inaction

- Idea \#7: Undertake an impossible task with a positive attitude

- Idea \#8: Think of some impractical things and then plan on doing them

- Idea \#9: Avoid anyone (even customers) if all they talk about is money

- Idea \#10: Innovate by ignoring what everyone else has done before you

- Idea \#11: Forget the past, especially your own company's successes ». (Sutton 2001) 